\title{
Entropy-Based Biomarkers for Individualized Re- sponse on Bosutinib Treatment in Chronic Phase CML
}

Technical report on the CML-modeling project, in collaboration with Pfizer, hosted on Vivli

Ali H. Esfahani

Joint Research Center for Computational Biomedicine (JRC-COMBINE)

RWTH Aachen University

Aachen, Germany 


\section{Introduction}

The invention of imatinib has turned chronic myeloid leukemia (CML), caused by the oncogenic fusion tyrosine kinase BCR-ABL, from a fatal blood cancer into a manageable disease. A follow-up study of 832 patients treated with imatinib reported $97.7 \%$ and $95.2 \%$ survival, for 6 and 8 years respectively [1]. The caveat of that publication, however, is that only the patients who were in complete cytogenetic response $(\mathrm{CCyR})$ after 2 years of imatinib treatment were considered. It has been shown that the CCyR for patients treated with imatinib as the first line of defense is $83 \%$, hence, there is a chance for the development of imatinib resistance, especially in the early stages of treatment [2]. Multiple drugs have been developed as the next generation of tyrosine kinase inhibitors (TKI) to replace imatinib in resistant patients. An example of such drugs is bosutinib, which is a second generation TKI that is safe and active in patients with resistance or intolerance to imatinib [3].

The phase III clinical trial of bosutinib demonstrated a higher major molecular response (MMR) rate at 12 months, faster times to CCyR and MMR, fewer ontreatment transformations to accelerated/blast phase, and fewer CML-related deaths with bosutinib, all compared to imatinib. However, the trial did not meet its primary endpoint of CCyR at 12 months [4]. In addition, adverse events with bosutinib versus imatinib were significantly more common for certain gastrointestinal events (diarrhea, vomiting, alanine aminotransferase and aspartate aminotransferase elevations, and pyrexia) [5]. In TKI clinical trials, such as the one for bosutinib, it is common to stratify patients into different risk groups using prognostic models such as Sokal, Euro, EUTOS, Hasford, ... [6-9]. Precise risk estimation is not only necessary for comparative assessment of different results, but more importantly, optimal risk and treatment selection necessitate accurate risk modeling. That is why improving risk estimation in CML is an ongoing effort, resulting in better scores such as the recent ELTS score $[10,11]$.

The common risk analysis scores are valid prognostication tools, each fulfilling specific purposes. Most of them use simple linear models that use few variables (e.g., age, spleen size, and blood cell counts). Such an approach is beneficial since these types of models are easy to use and have been adopted by the community. Yet, we argue that using a more sophisticated approach, for example integrating hematopoiesis and cancer dynamics models with patient biopsy omics to make a hybrid approach, can increase the efficacy of risk modeling.

In our previously published manuscript, "we show(ed) that combined population 
dynamic modeling and CML patient biopsy genomic analysis enables patient stratification at unprecedented resolution" [12]. In this project, we aim to use the same approach on BELA study data, accessed via Vivli platform [13, 14], to achieve an improved risk assessment for the patients. By applying a population dynamic based model, more specifically, the CML hematopoiesis model developed by Dingli and coworkers [15], combined with the entropy mapping of gene expression [12] utilizing the GEO's ${ }^{1}$ CML gene expression data set GSE4170² [16], we aim to build a new predictive model (Fig.1). Using this model, we separate the chronic phase (CP) of CML into different stages, thereby substratifying patients more precisely according to their disease progression and risk for transition into accelerated phase and succeeding blast crisis. Subsequently, we will analyze the clinical covariates available in the BELA study to identify non-gene expression-based substitute biomarkers for patient risk prediction, as well as the assessment of the likelihood of resistance towards specific TKIs associated with the intrinsic CP disease progression stage.

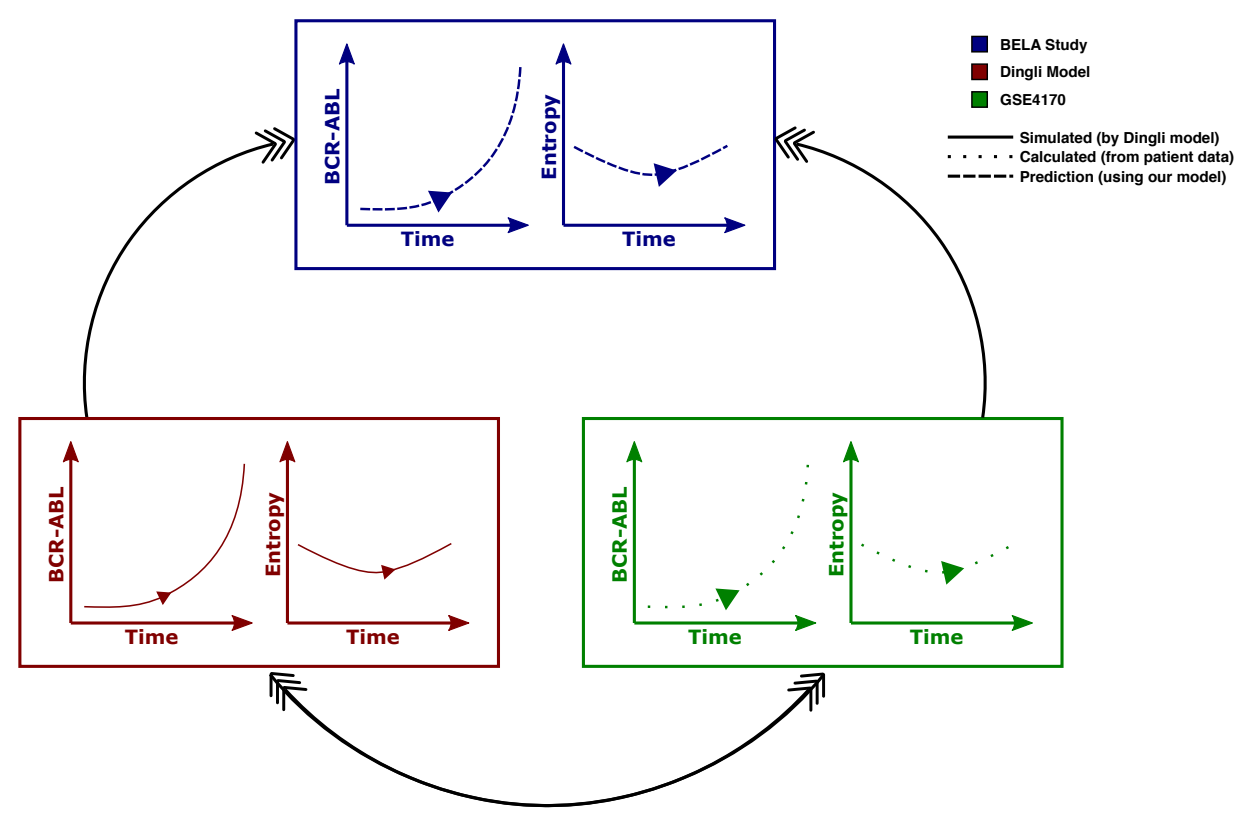

Figure 1: Combining Dingli hematopoiesis model (population dynamics) and GSE4170 data set (CML patient biopsy genomics) for prognostic modeling in patients in BELA trial.

\footnotetext{
${ }^{1}$ NCBI's Gene Expression Omnibus, accessible via https://www.ncbi.nlm.nih.gov/geo

${ }^{2}$ https://www.ncbi.nlm.nih.gov/geo/query/acc.cgi?acc=GSE4170
} 


\section{Results}

\section{Population Dynamic Model Implementation and}

\section{Benchmarking}

We implemented the hierarchically structured model of Dingli et al. [15] to simulate the hematopoietic dynamics in healthy and CML patients. To validate the correctness of the implemented model for our use-case, we calculated the ratio of blast cells through the development of CML (in the 6 years time window after the first BCR$\mathrm{ABL}$ mutation) in the peripheral blood using the model and compared it against values of patient samples reported in GSE4170. There is no "time from first mutation" value in the patient data set, hence, for making simulated values comparable to the measured ones, we calculated the similarity of the patient biopsies to CD34 ${ }^{+}$cells using PhysioSpace [17] and used it as a surrogate for the disease progression time (For a more detailed explanation, we recommend the original paper about this method [12]). Comparing the simulated values to the patient samples, it is evident that the model correctly simulates the dynamics of CML (Fig.2).
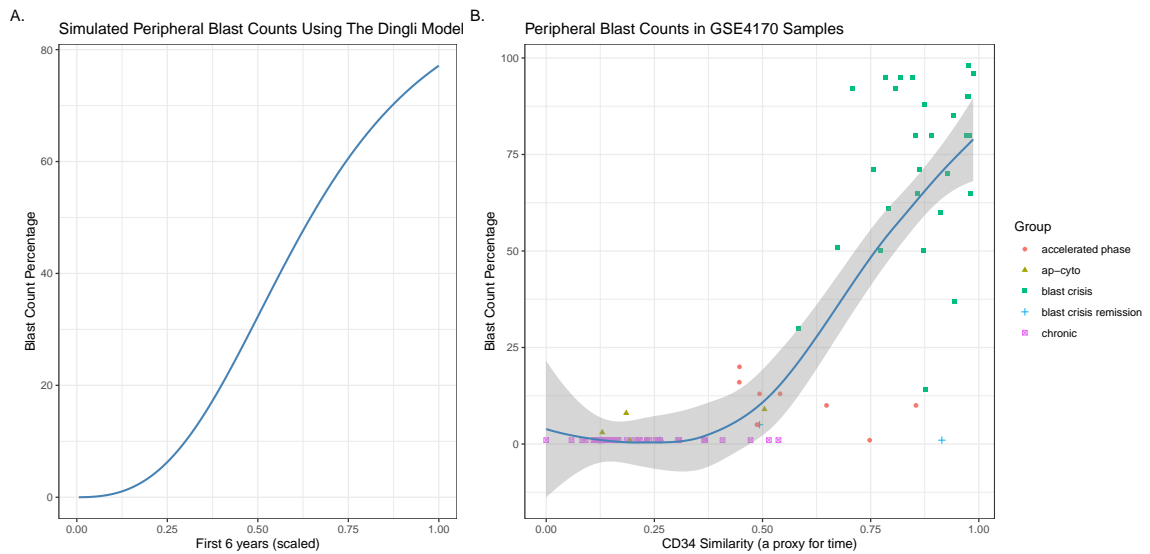

Figure 2: Blast count percentage in GSE4170 data set versus the simulated percentages from Dingli model (LOESS regression is used in panel B to represent the trend). 


\section{Progression Estimation in BELA Patients}

In the last section, we used the Dingli et al. model to estimate the blast cell count given the amount of time since BCR-ABL mutation. Based on the model, the blast count is a bijective function of time in CML (i.e., the blast count and the passed time have a one-to-one relationship). For this reason, one can use the reverse of this function to estimate the CML progression (i.e., the time from the first BCR-ABL mutation) from CML biomarkers such as peripheral blood blast count or BCR-ABL ratio (Fig.S1).

Using the population dynamic model, we stratified patients into low-progression (i.e. early stage of chronic CML, or low-risk) and high-progression (i.e. late stage of chronic CML, or high-risk), and compared this risk prognosis with the actual outcome in a retrospective analysis. Multiple CML outcome parameters are provided in BELA, in some of which the population dynamic model successfully estimated the risk. For instance, using the "time to major molecular response (MMR)" as a surrogate for the outcome or overall survival, patients that are estimated to be less progressed reach MMR significantly faster (one-sided $t$-test $p$ value of less than 0.001 ) than patients that are predicted to be in the later stages of chronic CML (Fig.3A).

\section{Molecular Response}

A.

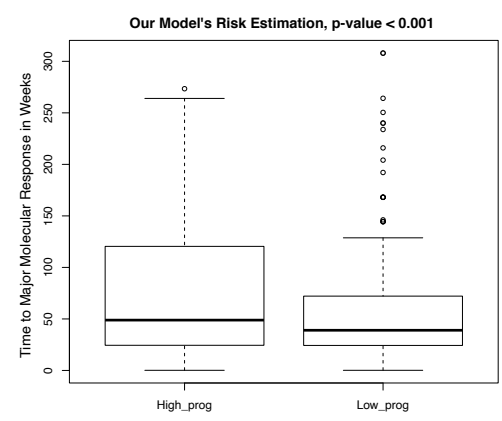

B.

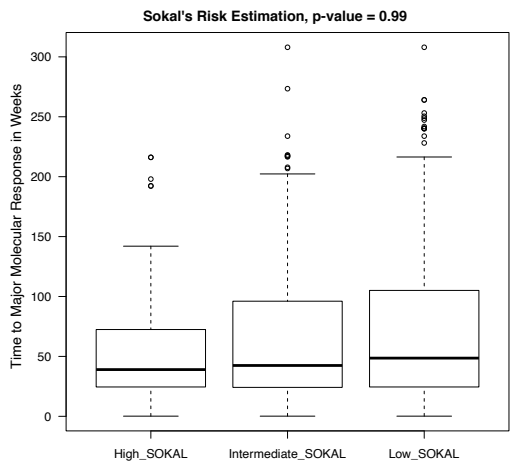

Figure 3: Time required to reach major molecular response in different risk groups. Risk is assessed using our model (panel A) and Sokal score (panel B). Reported $p$ values are from one-sided $t$-test, expecting low-risk groups to reach major molecular response significantly faster.

Compared to the population dynamic model risk prediction, Sokal risk estimation perform poorly in predicting molecular response in BELA patients. Sokal predicts higher-risk patients to reach MMR faster than lower-risk patients, which is contrary to what we expect as a risk prognostic score (Fig.3B). In addition, the one-sided $t$-test 
$p$ value for Sokal risk prediction between low- and high-risk groups is $\simeq 0.99$ (Bear in mind that we didn't use a two-sided $t$-test, i.e., we didn't test to see if the time to MMR is significantly different between low-risk and high-risk patients, but used a one-sided $t$-test, i.e., we tested to see if the time to MMR is significantly lower in low-risk patients versus high-risk patients.).

We should also mention that while we only compare our model to Sokal score here, contrasting our model to more recent scores, such as the European LeukemiaNet recommended ELTS [18], would have provided a better benchmarking versus the state-of-the-art. Unfortunately, the only reported risk score in BELA data set was Sokal. Furthermore, spleen size is not included in the data set, which is required if we intended to calculate ELTS, Euro, or EUTOS score ourselves.

Our risk estimation didn't perform well in all CML outcome parameters provided in BELA. For instance, predicting the cytogenic response was problematic for the population dynamic model. Assuming the "duration of cytogenic response" as a cytogenic response biomarker, population dynamic model high- and low-risk patient groups doesn't have a significantly different response ( $t$-test $p$ value of 0.33 ) (Fig.4A).

\section{Cytogenic Response}

A.

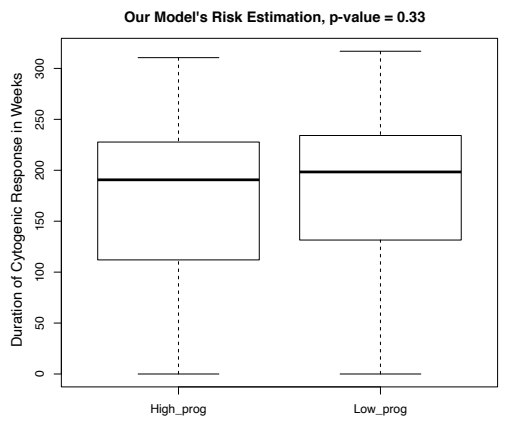

B.

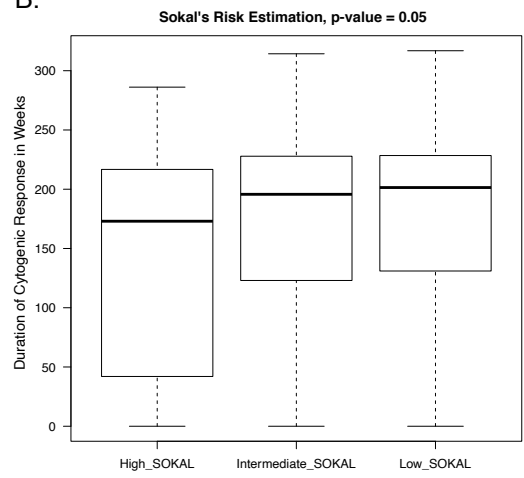

Figure 4: Duration of cytogenic response in different risk groups. Risk is assessed using our model (panel A) and Sokal score (panel B). Reported $p$ values are from one-sided $t$-test, expecting low-risk groups to keep cytogenic response for a longer time.

Sokal score predictions were marginally better for cytogenic responses. With a $p$ value of 0.05 , high Sokal score patients have a shorter cytogenic response and vice versa (Fig.4B). 


\section{Linking The Population Dynamic Model to CML Gene Expression for Improving the Efficacy}

As shown in the last section, the prognostic performance of Dingli's population dynamic model is limited, and in some cases inferior to basic models like Sokal score. In order to boost its performance, we integrated a CML gene expression data set with the population dynamic model, as published previously by Brehme et al. [12]. We utilized GSE4170 data set, which includes gene expression in 91 cases of CML in chronic (42 cases), accelerated (17 cases), and blast phases (32 cases). By using PhysioSpace method [17] to find the similarities between the samples, we combine the mechanistic model and the patient data, and use the resulted hybrid model to estimate gene expressions of patient samples in BELA trial.

As shown previously [12], the entropy of CML gene expression displays a nonmonotonic dynamic trend with a singular minimum. Using the simulated gene expression for BELA patients, we calculated the entropy to better pinpoint the CML progression level in each patient, and consequently have a better risk estimation. In figure 5, we plotted the patient entropies and compared them to the surrogate progression values from cytogenic and molecular responses, more specifically, duration of $\mathrm{CCyR}$ and time to MMR.

A.

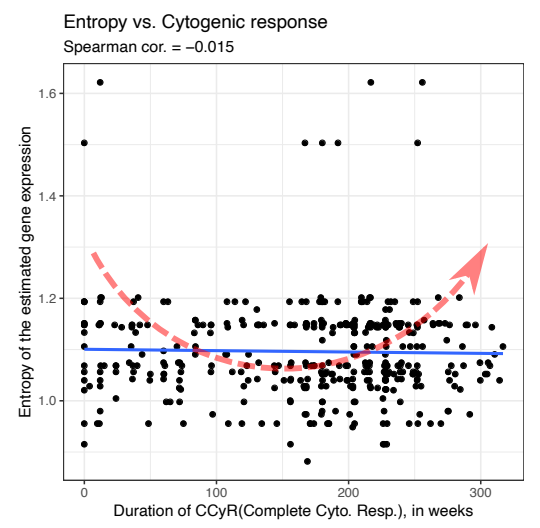

B.

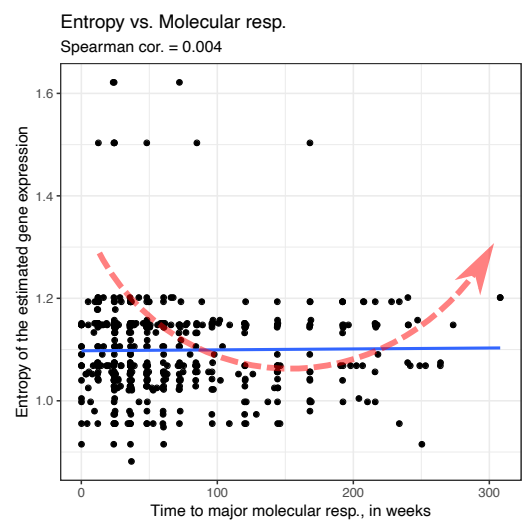

Figure 5: Expected vs actual behavior of gene expression entropy in BELA patients.

The expected behavior of the entropy values in figure 5 is plotted with a dashed red arrow, while the calculated values are marked using black dots, with a linear fitted line in blue. As evident in the figure, entropy values don't have the expected non- 
monotonic dynamic trend, therefore, the patient stratification approach of Brehme and co-workers [12] was not applicable to the patients in the BELA study.

\section{Black Box Modeling Scheme}
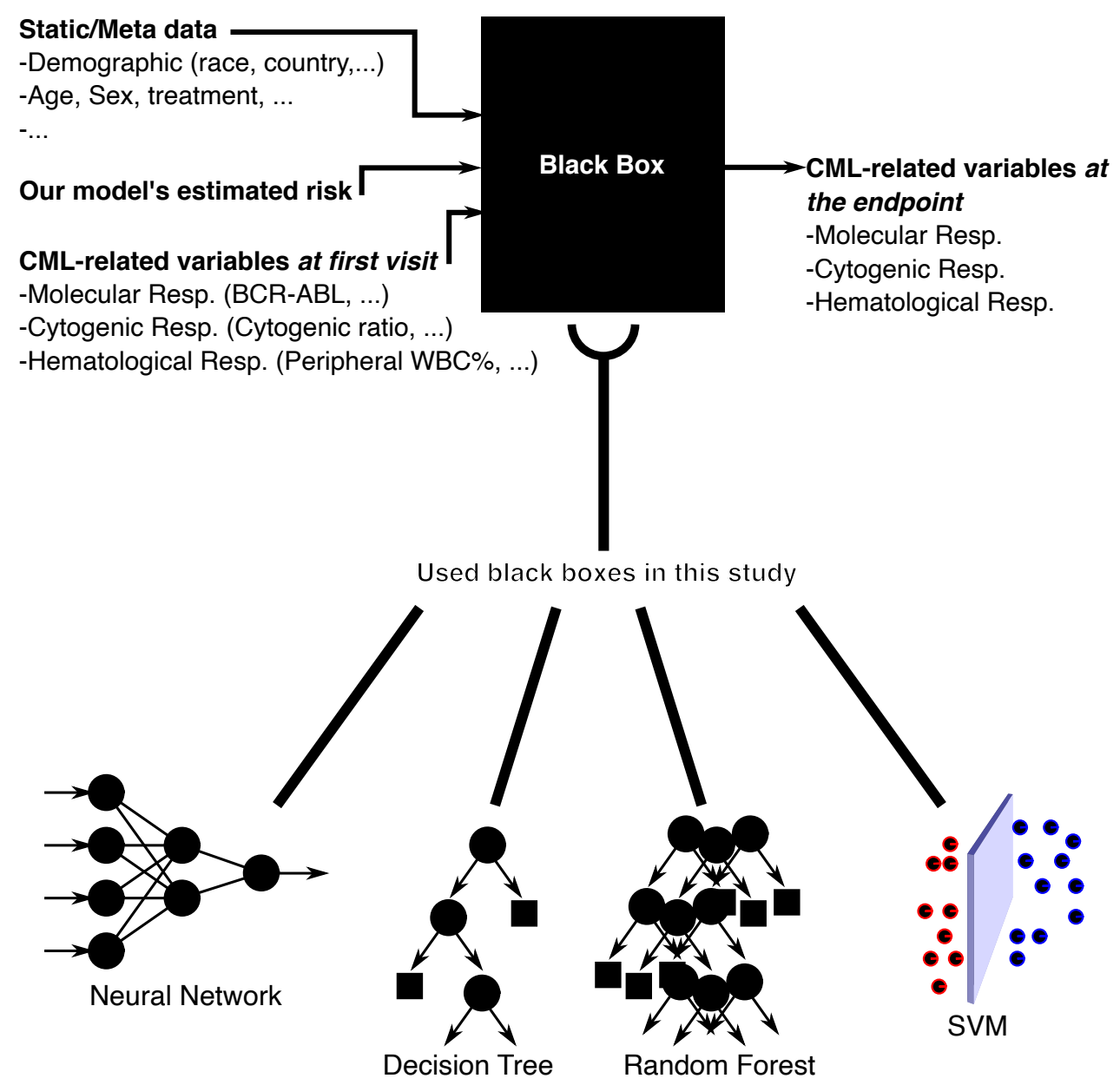

Figure 6: Black Box Strategy. 


\section{Combining Clinical Covariates Using a Black Box Modeling Approach}

Another objective of this project was to analyze the clinical covariates in the BELA study, identify CML-relevant variables, and ultimately improve the risk prediction. To achieve this objective, we used black box modeling to simultaneously detect and choose relevant covariates, while fitting a better model for patient stratification and risk estimation.

For the black box, we employed a neural network (shallow, i.e., with one hidden layer), decision tree, random forest, and support vector machine (SVM). For each black box, we used available static data in BELA (e.g., age, sex, ...), the output of the population dynamic model, and CML-related measures (molecular, cytogenic, and hematological response variables) at the first visit, i.e., time $=0$ to predict the endpoint values for the CML-related measures (Fig.6).

Based on the results, the black boxes could not perform better than the models from the last sections (Table 1). Analyzing the importance of input variables for each black box explains this mediocre performance: in most cases, models fit on BCR, $\mathrm{ABL}$, or a combination such as $\mathrm{m}$ _ratio (which is the normalized BCR-ABL ratio), which makes their performance comparable to the population dynamic model, which is mainly a function of BCR-ABL ratio.

In table 1 , the reported $p$ values are from the $t$-test of the black boxes output between high- and low-risk patients. Although in some cases, such as the SVM's $p$ value $<10^{-4}$, it seems the risk prediction performance is adequate, using other assessment methods points toward a mediocre prognostic performance. For instance, for the SVM black box, the AUC (area under the curve) of ROC (receiver operating characteristic) curve is $\approx 0.6$.

\begin{tabular}{||cllll||}
\hline & $\begin{array}{l}\text { Neural Net- } \\
\text { work }\end{array}$ & $\begin{array}{l}\text { Decision } \\
\text { Tree }\end{array}$ & SVM & $\begin{array}{l}\text { Random } \\
\text { Forest }\end{array}$ \\
\hline \hline Important Inputs & - & $\begin{array}{l}\text { treatment, } \\
\text { BCR, ABL, } \\
\text { m_ratio }\end{array}$ & $\begin{array}{l}\text { Our } \\
\text { Model's } \\
\text { risk, ratioF, } \\
\text { m_ratio }\end{array}$ & $\begin{array}{l}\text { BCR, ABL, } \\
\text { m_ratio }\end{array}$ \\
\hline p value & 0.0016 & 0.1 & $<10^{-4}$ & 0.001 \\
\hline
\end{tabular}

Table 1: Black Box Performances. 


\section{Discussion}

In this project, we explored a hybrid approach for stratifying CML patients in BELA trial. The aim of the project was two-fold: firstly, to classify the CML patients into different clusters of "risk", and secondly, to analyze the clinical variables in BELA in concordance with the discovered risk clusters to discover new biomarkers.

Our approach was proven advantageous in some cases. The population dynamic model could outperform other common risk estimators (i.e., Sokal score). That said, in some other cases, our method performed similarly to the state-of-the-art. For instance, locating the exact CML disease progression level in the chronic phase was carried off by utilizing the entropy of gene expression levels in previous work. However, applying the same approach was not beneficial for stratifying the patients in BELA.

A potential reason for the handicapped performance of our model is the assumptions that are not met in this project's use case. For example, Dingli's hematopoiesis model simulates the CML disease progression without considering any external intervention, while all patients in BELA trial are treated with TKIs. Another reason could have stemmed from the gene expression estimation we performed in this project: gene expression was not among the measured variables in BELA study, and since our model needed the expression levels for a proper risk assessment, we estimated the expression values by mapping BELA patient samples to a CML gene expression data set. We reckon that this projection could have introduced errors into the model.

Overall, we presented a variety of novel risk assessment methods in this project. Though each approach had its pros and cons, we are of the opinion that our hybrid approaches, which can take advantage of external data and models by integrating them into the method, can pave the way for constructing better CML prognostic methods in the future.

\section{Acknowledgements}

This publication is based on research using data from Pfizer Inc. (study ID: NCT00574873 / B1871008) that has been made available through Vivli, Inc. Vivli has not contributed to or approved, and is not in any way responsible for, the contents of this publication. 


\section{Bibliography}

[1] Carlo Gambacorti-Passerini et al. "Multicenter independent assessment of outcomes in chronic myeloid leukemia patients treated with imatinib." In: Journal of the National Cancer Institute 103.7 (2011), pages 553-561.

[2] Roberta Bitencourt, Ilana Zalcberg, and Iúri Drumond Louro. "Imatinib resistance: a review of alternative inhibitors in chronic myeloid leukemia." In: Revista brasileira de hematologia e hemoterapia 33 (2011), pages 470-475.

[3] TH Bruemmendorf et al. "Bosutinib is safe and active in patients (pts) with chronic phase (CP) chronic myeloid leukemia (CML) with resistance or intolerance to imatinib and other tyrosine kinase inhibitors." In: Journal of Clinical Oncology 26.15_suppl (2008), pages 7001-7001.

[4] Jorge E Cortes et al. "Bosutinib versus imatinib in newly diagnosed chronicphase chronic myeloid leukemia: results from the BELA trial." In: Journal of Clinical Oncology 30.28 (2012), page 3486.

[5] Carlo Gambacorti-Passerini et al. "Safety of bosutinib versus imatinib in the phase 3 BELA trial in newly diagnosed chronic phase chronic myeloid leukemia." In: American journal of hematology 89.10 (2014), pages 947-953.

[6] Markus Pfirrmann et al. "Prognostic scores for patients with chronic myeloid leukemia under particular consideration of competing causes of death." In: $A n$ nals of hematology 94.2 (2015), pages 209-218.

[7] Joseph E Sokal et al. "Prognostic discrimination in" good-risk" chronic granulocytic leukemia." In: (1984).

[8] Joerg Hasford et al. "A new prognostic score for survival of patients with chronic myeloid leukemia treated with interferon alfa Writing Committee for the Collaborative CML Prognostic Factors Project Group." In: JNCI: Journal of the National Cancer Institute 90.11 (1998), pages 850-859.

[9] Joerg Hasford et al. "Predicting complete cytogenetic response and subsequent progression-free survival in 2060 patients with CML on imatinib treatment: the EUTOS score." In: Blood, The Journal of the American Society of Hematology 118.3 (2011), pages 686-692.

[10] Markus Pfirrmann et al. "Prognosis of long-term survival considering diseasespecific death in patients with chronic myeloid leukemia." In: Leukemia 30.1 (2016), pages 48-56. 
[11] Markus Pfirrmann et al. "The EUTOS long-term survival (ELTS) score is superior to the Sokal score for predicting survival in chronic myeloid leukemia." In: Leukemia 34.8 (2020), pages 2138-2149.

[12] Marc Brehme et al. "Combined population dynamics and entropy modelling supports patient stratification in chronic myeloid leukemia." In: Scientific reports 6.1 (2016), pages 1-12.

[13] Barbara E Bierer et al. "A global, neutral platform for sharing trial data." In: New England Journal of Medicine 374.25 (2016), pages 2411-2413.

[14] Rebecca Li et al. "Timely access to trial data in the context of a pandemic: the time is now." In: BMJ open 10.10 (2020), e039326.

[15] David Dingli, Arne Traulsen, and Jorge M Pacheco. "Chronic myeloid leukemia: origin, development, response to therapy, and relapse." In: Clinical Leukemia 2.2 (2008), pages $133-139$.

[16] Jerald P Radich et al. "Gene expression changes associated with progression and response in chronic myeloid leukemia." In: Proceedings of the National Academy of Sciences 103.8 (2006), pages 2794-2799.

[17] Michael Lenz et al. "PhysioSpace: relating gene expression experiments from heterogeneous sources using shared physiological processes." In: PLoS One 8.10 (2013), e77627.

[18] Andreas Hochhaus et al. "European LeukemiaNet 2020 recommendations for treating chronic myeloid leukemia." In: Leukemia 34.4 (2020), pages 966-984. 


\section{Supplemental Material}

Figures

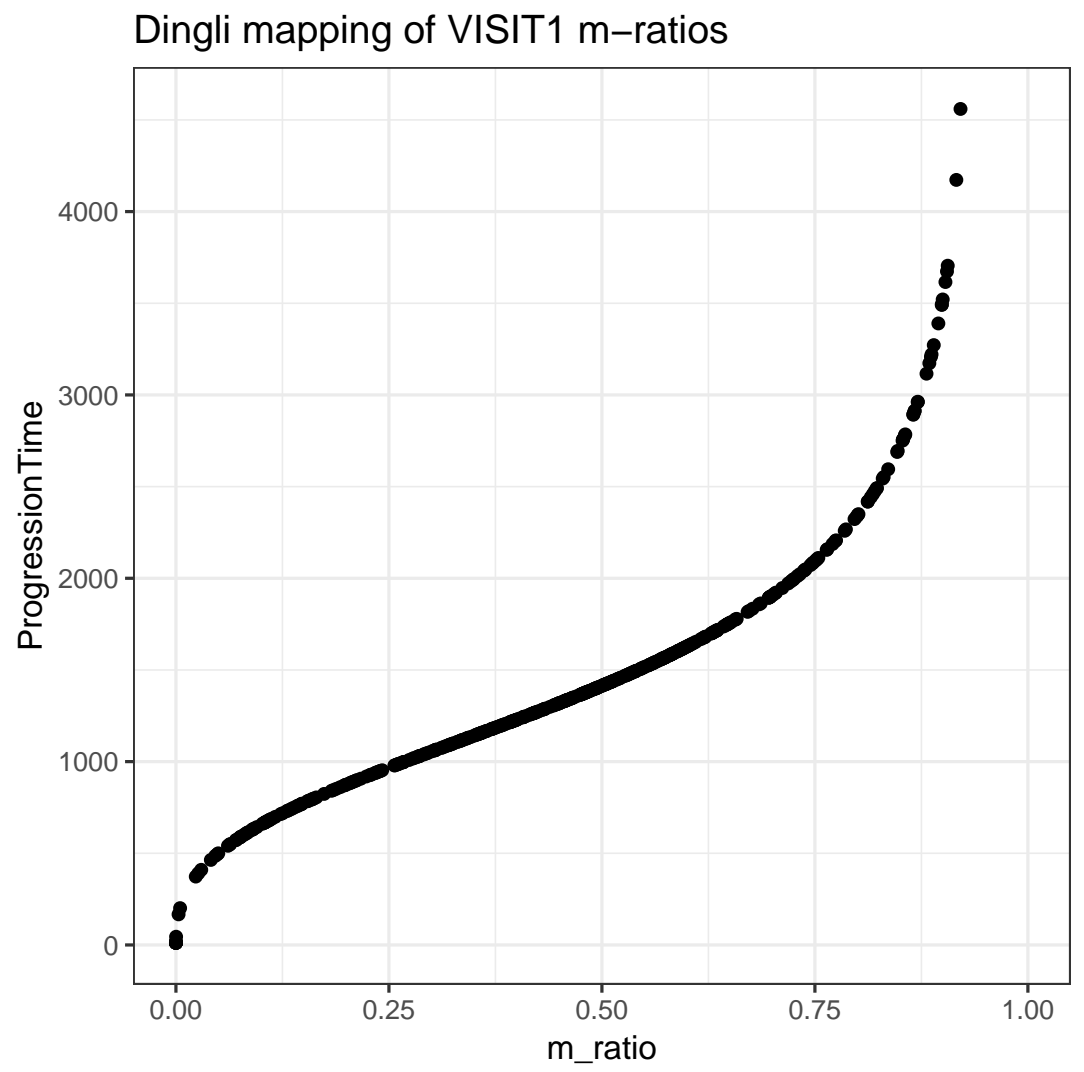

Figure S1: Estimating CML progression level from m-ratio (BCR-ABL/ABL). This approach only works for non-treated patients, i.e., only in first visit $(\mathrm{VISIT}=1)$ for BELA patients. 